\title{
Targeting hexokinase 2 inhibition promotes radiosensitization in HPV16 E7-induced cervical cancer and suppresses tumor growth
}

\author{
YUAN LIU ${ }^{1}$, TRACY MURRAY-STEWART ${ }^{2}$, ROBERT A. CASERO $\mathrm{Jr}^{2}$, IOANNIS KAGIAMPAKIS ${ }^{2}$, \\ LIHUA JIN $^{3}$, JIAWEN ZHANG ${ }^{4}$, HUIHUI WANG $^{5}$, QI CHE ${ }^{1}$, HUAN TONG $^{1}$, JIEQI KE ${ }^{1}$, \\ FEIZHOU JIANG ${ }^{1}$, FANGYUAN WANG ${ }^{1}$ and XIAOPING WAN ${ }^{6}$
}

\author{
${ }^{1}$ Department of Obstetrics and Gynecology, Shanghai General Hospital, Shanghai Jiaotong University School of Medicine, \\ Shanghai, P.R. China; ${ }^{2}$ Sidney Kimmel Comprehensive Cancer Center, Johns Hopkins University School of Medicine, \\ Baltimore, MD; ${ }^{3}$ Genomic Medicine Institute, Lerner Research Institute, Cleveland Clinic, Cleveland, OH, USA; \\ ${ }^{4}$ Department of Obstetrics and Gynecology, Shanghai Tenth People's Hospital, Shanghai Tongji University; \\ ${ }^{5}$ Department of Obstetrics and Gynecology, International Peace Maternity and Child Health Hospital \\ Affiliated with Shanghai Jiao Tong University; ${ }^{6}$ Department of Obstetrics and Gynecology, \\ Shanghai First Maternity and Infant Hospital, Tongji University School of Medicine, Shanghai, P.R. China
}

Received February 14, 2017; Accepted April 13, 2017

DOI: 10.3892/ijo.2017.3979

\begin{abstract}
In order to improve the sensitivity of cervical cancer cells to irradiation therapy, we targeted hexokinase 2 (HK2), the first rate-limiting enzyme of glycolysis, and explore its role in cervical cancer cells. We suppressed HK2 expression and/ or function by shRNA and/or metformin and found HK2 inhibition enhanced cells apoptosis with accelerating expression of cleaved PARP and caspase-3. HK2 inhibition also induced much inferior proliferation of cervical cancer cells both in vitro and in vivo with diminishing expression of mTOR, MIB and MGMT. Moreover, HK2 inhibition altered the metabolic profile of cervical cancer cells to one less dependent on glycolysis with a reinforcement of mitochondrial function and an ablation of lactification ability. Importantly, cervical cancer cells contained HK2 inhibition displayed more sensitivity to irradiation. Further results indicated that HPV16 E7 oncoprotein altered the glucose homeostasis of cervical cancer cells into glycolysis by coordinately promoting HK2 expression and its downregulation of glycolysis. Taken together, our findings supported a mechanism whereby targeting HK2 inhibition contributed to suppress HPV16 E7-induced tumor glycolysis metabolism phenotype, inhibiting tumor growth, and induced apoptosis, blocking the cancer cell energy sources and ultimately enhanced the sensitivity of HPV(+) cervical cancer cells to irradiation therapy.
\end{abstract}

Correspondence to: Professor Xiaoping Wan, Department of Obstetrics and Gynecology, Shanghai First Maternity and Infant Hospital, Tongji University School of Medicine, 391 Yaohua Road, Pudong, Shanghai 200040, P.R. China

E-mail: wanxiaoping@tongji.edu.cn

Key words: hexokinase 2, HPV16 E7, cervical cancer, glycolysis, irradiation

\section{Introduction}

Cervical cancer is one of the most common cancers in women worldwide and is especially prevalent in developing countries. For example, $\sim 98,900$ new cases of cervical cancer and 30,500 deaths were reported in China in 2015 (1). Historically, surgery and radiotherapy (RT) have been the two major treatments for invasive cervical cancer. Most women with metastatic cervical cancer or local recurrence after radiotherapy are candidates for palliative chemotherapy. Radiotherapy is a pre- or postoperative adjuvant or primary treatment in most locally advanced cervical cancers. However, the resistance of tumor cells to radiation is a major therapeutic problem.

Most normal tissues metabolize the 6-carbon glucose into the 3-carbon pyruvate and then exploit the resulting energy in the form of ATP via 'oxidative phosphorylation' (OXPHOS) in the mitochondria. In contrast, cancer cells primarily use aerobic glycolysis to convert glucose into lactic acid at a high rate to support growth, even in the presence of oxygen. This metabolic alteration is referred to as the 'Warburg effect' and it is this energy metabolism that fuels tumor cell growth and division, including chronic and often uncontrolled cell proliferation, and may facilitate apoptosis resistance $(2,3)$. A critical player in this frequent cancer metabolism phenotype is the mitochondrial-bound hexokinase 2 (HK2), the enzyme that catalyzes the first rate-limiting step of the glycolytic pathway, where glucose is phosphorylated to glucose-6-phosphate (G-6-P) with ATP consumption (4). The relatively high expression of HK2 in cancer cells is responsible for the accelerated glucose flux (5) and can distinguish malignant cells from the normal cells, and contributes to tumor initiation, maintenance and metastasis (6-8). Upon a key oncogenic AKT pathway activation, HK2 translocates to the mitochondrial outer membrane, where it interacts with the voltage-dependent anion channel (VDAC) to help mitochondrion escape strong product inhibition by G-6-P and obtain priority access to newly 
synthesized ATP (9-11). Moreover, HK2 eventually inhibits caspase-9-dependent apoptosis, by blocking the release of cytochrome $c$ and interacting with the permeability transition pore including VDAC1 and Bax (11-14). Thus, HK2 not only improves the malignant cells' energy supply by making them more dependent on the glycolytic metabolic profile and more adaptive to survive in an anoxic environment, it also immortalizes and protects malignant cells against apoptosis through direct interaction with mitochondria.

The responses of malignant tumors to irradiation vary in their respective resistance mechanisms. Radioresistance can be affected by a lack of oxygen (15), cell cycle status (16), DNA damage and repair (17), apoptosis (18), growth factors and oncogenes (19), stem cells (20), and other factors. Among these, hypoxia-related radioresistance is the most important. The hypoxic microenvironment can potentially serve as a protective sheath against tumor damage $(15,21-23)$. Glycolysis, which is the main metabolic profile for tumor cells according to Warburg effect, is also closely related with radioresistance (24-27). Reports have proven that inhibition of the Warburg effect enhances the radiosensitivity of cancers (28-30). Some squamous cell carcinomas, such as cervical squamous cell carcinoma, have proven to be the most modifiable type of tumor cells by the manipulation of hypoxia in practice as they are more likely to maintain colony formation potential during long-term hypoxia. Although a number of recent trials investigating hypoxic modifications have displayed considerable efficacy, the effect has been too limited to raise a broader interest in this field. Thus, in seeking a breakthrough in providing an appropriate application for cervical cancer treatment with superior irradiation sensitivity, we aimed to exploit hypoxic glycolytic metabolism as a property unique to tumor cells, with a focus on hexokinase 2 (HK2), the essential regulatory point of the glycolysis pathway.

\section{Materials and methods}

Ethics. All applicable international and institutional guidelines for the care and use of animals were followed. Animal experiments were performed in strict accordance with the Guide for the Care and Use of Laboratory Animals and were approved by the Department of Laboratory Animal Science at Shanghai Jiao Tong University School of Medicine. This report does not contain any studies with human participants performed by any of the authors.

TCGA data. Level 3 normalized counts of HK2 (RNA-Seq; Illumina) data and cervical cancer clinical data were downloaded from TCGA and analyzed in the R statistical environment. Survival rates were calculated using the Kaplan-Meier method and the log-rank test was used to compare the survival curves. The heatmap of the HK2-normalized counts from level 3 RNA-Seq TCGA data was made by Excel: the green color is aligned to the largest normalized count and the deepest red is aligned to the smallest normalized count.

Cell lines and cell culture. The human cervical carcinoma lines HPV16(+) SiHa, HPV18(+) HeLa, HPV18(+) SW756 and HPV(-) C33A were purchased from ATCC and maintained in Dulbecco's modified Eagle's medium (DMEM) F-12 1:1 medium (Gibco) with 10\% fetal bovine serum (FBS; Gibco), $100 \mathrm{U} / \mathrm{ml}$ penicillin, sodium pyruvate and L-glutamine in a humidified atmosphere of $5 \% \mathrm{CO}_{2}$ at $37^{\circ} \mathrm{C}$. The cell lines were maintained in the laboratory of Dr S.F. Wu for eight months and no further authentication was performed. Hypoxia environment was made by treating cells with $\mathrm{CoCl}_{2}$ (Sigma, $150 \mu \mathrm{M} / \mathrm{l})$ for $24 \mathrm{~h}(31-33)$.

Plasmid and lentivirus transfections. The overexpression plasmids, including pCAG-myc-HPV16 E6, pCAG-mycHPV16 E7, and pCAG-myc-blank, were obtained from S.F. Wu, and the HK2 and HIF-1 $\alpha$ shRNAs were purchased from Shanghai GenePharma Co. Ltd. Plasmid transfection was performed as previously described (34). Lentiviruscarrying small hairpin RNAs (shRNA) targeting HK2 and HIF-1 $\alpha$ were transfected into cells at $60 \%$ confluence in 6 -well plates for infection with polybrene $(5 \mu \mathrm{g} / \mathrm{ml}$; GenePharma). Medium was refreshed after $24 \mathrm{~h}$ of transfection and the cells were incubated another $72 \mathrm{~h}$ before analysis of mRNA or protein expression. The sequence used to generate the shRNA targeting HK2 is 5'-GGGTGAAAGTAACGGACAATG-3'. The sequence used to generate the shRNA targeting HIF-1 $\alpha$ is 5'-GCCGAGGAAGAACTATGAACA-3'.

Western blot analysis and RT-PCR. For western blots, briefly, $60 \mu \mathrm{g}$ of protein was separated by SDS-PAGE and transferred onto polyvinylidene fluoride (PVDF) membranes. Membranes were incubated with blocking buffer for $2 \mathrm{~h}$ followed by incubation for $15 \mathrm{~h}$ with the following primary antibodies: anti-PARP (diluted at 1:500; Cell Signaling Technology, Beverley, MA, USA, \#5625), anti-HPV16 E7 (diluted at 1:100; Bioss, Shanghai, China, \#bs-10446R), anti-caspase 3 (diluted at 1:500; Cell Signaling Technology, \#9664), antiBcl2 (diluted at 1:500; Antibody Revolution, CA, USA, \#ARH2043), anti-GAPDH (Epitomics), anti-HK2 (diluted at 1:500; Aviva Systems Biology, CA, USA, \#ARP54303_P050), and anti-HIF-1 $\alpha$ (diluted at 1:100; Boster, Wuhan, China, \#PB0245). An additional hour of incubation was performed with the appropriate secondary antibody. For RT-PCR, total RNA was extracted with TRIzol (Invitrogen). cDNA was synthesized from $1 \mu \mathrm{g}$ of total RNA according to the Takara protocol. The genes of interest were amplified using appropriate primers with 40 cycles. The primers sequences are listed in Table I.

Flow cytometric analysis of apoptosis. For the apoptosis assay, the cells were trypsinized and washed with fresh medium. Cells were then centrifuged for $3 \mathrm{~min}$ at 1,500 rpm and the supernatant was discarded. The cell pellets were resuspended in $1 \mathrm{X}$ binding buffer at $1-5 \times 10^{6} / \mathrm{ml}$ and stained for $15 \mathrm{~min}$ using the Annexin V-PE/7AAD Apoptosis kit (eBioscience, CA, USA), according to the manufacturer's instructions. The number of apoptotic cells was analyzed by flow cytometry (BD Accuri C6, USA).

Cell metabolism assays. Glycolytic rates were measured by calculating extracellular acidification (ECAR) and oxygen consumption rates (OCR) simultaneously in real-time using the Seahorse Biosciences Extracellular Flux Analyzer. Cervical cancer cells $\left(4 \times 10^{5}\right)$ were seeded into XF 24-well cell 
Table I. Primers used in this study.

\begin{tabular}{ll}
\hline Primer & \multicolumn{1}{c}{ Sequence $\left(5^{\prime} \rightarrow 3^{\prime}\right)$} \\
\hline HK2 F & TGCTTGCCTACTTCTTCACG \\
HK2 R & CATCTGGAGTGGACCTCACA \\
E6 F & CGACCCAGAAAGTTACCACAGT \\
E6 R & AATCCCGAAAAGCAAAGTCATA \\
E7 F & GAGGAGGAAGATGAAATAGATGG \\
E7 R & AACCGAAGCGTAGAGTCACAC \\
Glut1 F & AATTTCATTGTGGGCATGTG \\
Glut1 R & TCCTCGGGTGTCTTGTCACT \\
HIF-1 $\alpha$ F & GCAGCAACGACACAGAAACT \\
HIF-1 $\alpha$ R & GCAGGGTCAGCACTACTTCG \\
MGMT F & TGGAGCTGTCTGGTTGTGAG \\
MGMTR & GGGCTGCTAATTGCTGGTAA \\
GAPDH F & AGAAGGCTGGGGCTCATTTG \\
GAPDH R & AGGGGCCATCCACAGTCTTC \\
TFAM F & CGTTTCTCCGAAGCATGTG \\
TFAM R & TCCGCCCTATAAGCATCTTG \\
LDHA F & AGCCCGATTCCGTTACCTA \\
LDHA R & TGCTTGTGAACCTCTTTCCA \\
MIB F & GCGATGCTTCCAACTTTAGG \\
MIB R & TGCCCATTTACATCCACATC \\
mTOR F & CCTCACAAGACATCGCTGAA \\
mTOR R & GGATCTCCAGCTCTCCAAAGT \\
\hline &
\end{tabular}

culture micro-plates (Seahorse Biosciences) by BD Cell-Tak (BD Biosciences, Oxfordshire, UK), and plates were incubated at $37^{\circ} \mathrm{C}$ for $1 \mathrm{~h}$ before OCR and ECAR analysis. The experimental procedures included monitoring the cells for oxygen consumption and lactic acid production while injecting metabolic compounds into the media. The compounds used were D-glucose $(2 \mathrm{~g} / \mathrm{l})$, oligomycin $(1 \mu \mathrm{M})$, and 2-deoxyglucose $(100 \mathrm{mM})$, which provided glycolysis-associated ECAR, the maximum glycolytic capacity, and non-glycolytic ECAR, respectively. Seahorse Biosciences assay media, which is an unbuffered DMEM without glucose, pyruvate, or biocarbonate, was used during experimentation. We adjusted the $\mathrm{pH}$ of the media before each use with $\mathrm{HCL}$ and $\mathrm{NaOH}$. Data are presented as extracellular acidification rate (ECAR; $\mathrm{mpol} / \mathrm{min}$ ) for glycolysis and oxygen consumption rate (OCR: $\mathrm{pmol} / \mathrm{min}$ ) for oxidative phosphorylation. Each assay was performed in quadruplicate and representative data from three independent experiments are shown.

Nude mouse xenograft models. Female athymic nude mice at 6 weeks of age were purchased from the Shanghai Experimental Animal Center of the Chinese Academy of Science. For xenograft tumor formation assays, mice were randomly separated into two groups (five per group). SiHa cells transfected with shNC or shHK2 were subcutaneously injected into the two groups at a concentration of $1 \times 10^{7}$ cells per mouse. We measured the tumor size every week for five weeks. Then the mice were sacrificed, the tumors removed, fixed in formalin,
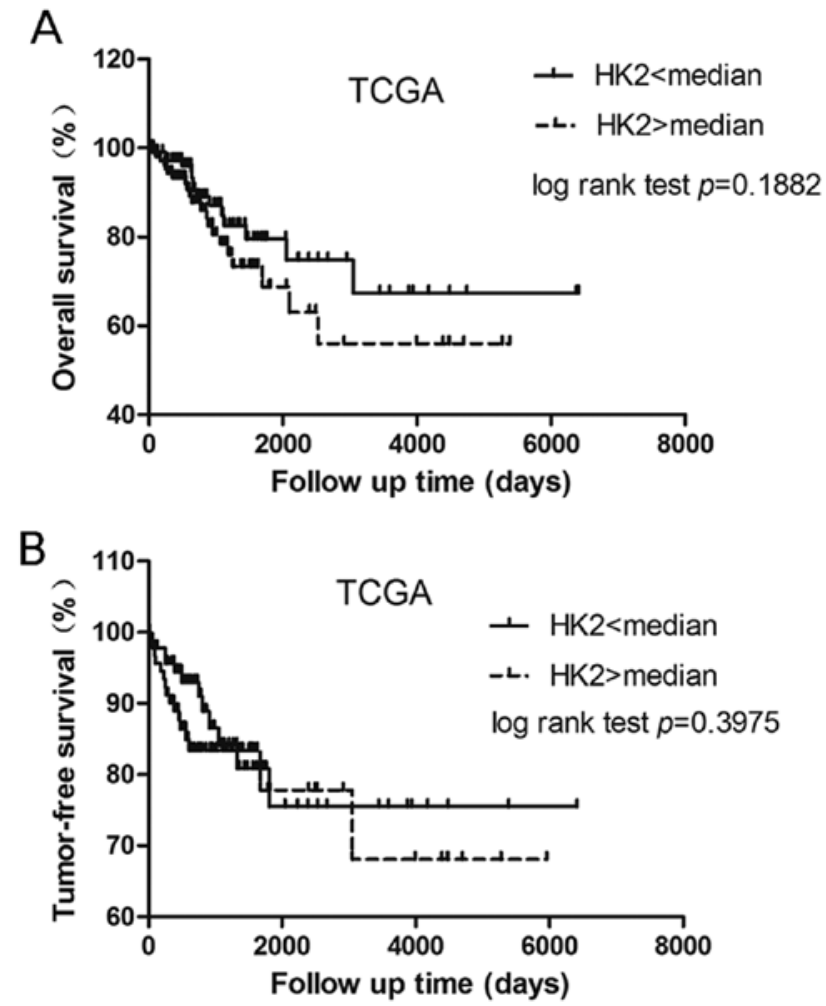

Figure 1. Overall survival and tumor-free survival between HK2 high expression group and HK2 low expression group from TCGA. Kaplan-Meier plot of the 236 cervical cancer patients according to normalized HK2 counts from TCGA RNA-Seq. Normalized counts of HK2 were dichotomized at the median and the cohort was divided into two groups with relatively low and high mRNA expression levels of HK2. Kaplan-Meier curves are shown for (A) overall survival and (B) tumor-free survival.

embedded in paraffin, and sectioned for IHC staining. The tumor volume was calculated as follows: tumor volume $\left(\mathrm{mm}^{3}\right)$ $=($ longest diameter $) \times(\text { shortest diameter })^{2} \times 0.5$.

Statistical analysis. The differences between groups in protein levels detected by western blots, RNA levels determined by RT-PCR, flow cytometric analyses, cell metabolism assays, and in vivo experiments were analyzed by Student's t-test. A two-sided test with $\mathrm{p}<0.05$ was considered statistically significant. All statistical analyses were performed using SAS Release 8.02 (SAS Institute Inc., Cary, NC, USA).

\section{Results}

Analysis of survival and HK2 expression level from TCGA data. First we investigated the relationship between HK2 expression and the corresponding patient prognosis. For each patient in the cervical cancer cohort $(n=234)$, a normalized RNA-Sequence count which stands for the mRNA expression of HK2, was calculated from TCGA data. Normalized counts were dichotomized at the median and the cohort was divided into two groups with relatively low and high expression levels of HK2. Curves for overall survival (OS) and tumor-free survival were plotted according to the Kaplan-Meier method, with p-values determined by the log-rank test. The difference between the two groups for overall survival and tumor-free survival was not statistically significant (Fig. 1). We also orga- 
HK2 mRNA expression in different HPV subtypes from TCGA

\begin{tabular}{|c|c|c|c|c|c|c|c|c|c|c|}
\hline \multirow[t]{18}{*}{ HPV16 } & 2164.745 & 1817.204 & 1346.818 & 1821.584 & 3455.653 & 1948.341 & 3185.587 & 1943.422 & 1427.785 & 1668.758 \\
\hline & 2562.395 & 2886.296 & 1263.036 & 6033.528 & 1738.794 & 2097.893 & 2367.288 & 1633.006 & 7091.513 & 3170.461 \\
\hline & 2659.852 & 2612.212 & 2928.838 & 2016.297 & 3246.571 & 3158.339 & 2253.429 & 3781.818 & 2492.558 & 757.9099 \\
\hline & 5860.942 & 1987.83 & 1935.721 & 1231.681 & 4624.11 & 2661.454 & 2702.186 & 4232.775 & 2884.654 & 2808.372 \\
\hline & 1531.816 & 1735.235 & 2241.265 & 2607.668 & 1873.549 & 1595.191 & 2200.583 & 3255.566 & 2815.556 & 2682.124 \\
\hline & 2060.691 & 2534.939 & 1805.245 & 3029.792 & 3219.085 & 1733.399 & 2045.234 & 1903.352 & 2272.896 & 1889.854 \\
\hline & 2020.298 & 1971.02 & 1709.043 & 1958.219 & 2336.172 & 2732.145 & 2231 & 1643.461 & 1793.159 & 2347.768 \\
\hline & 1481.904 & 2543.045 & 2107.908 & 3357.487 & 2548.497 & 3092.984 & 3314.246 & 2576.242 & 2543.993 & 3630.823 \\
\hline & 3042.308 & 3664.819 & 2236.797 & 2665.881 & 1617.422 & 2954.254 & 2078.947 & 2724. 188 & 2202.363 & 3342.719 \\
\hline & 2429.562 & 2000.775 & 1878.478 & 2852.071 & 1990.077 & 1691.522 & 2402.416 & 2542.26 & 2374.459 & 1477.408 \\
\hline & 2696.594 & 3186.4 & 2310.355 & 1751.129 & 2284.058 & 1620.557 & 2242.32 & 2729.069 & 2458.846 & 2116.969 \\
\hline & 2954.869 & 3106.656 & 1295.459 & 2542.281 & 2092.026 & 2042.113 & 2442.078 & 2215.356 & 3591.653 & 3132.547 \\
\hline & 2343.159 & 3884.037 & 2871.582 & 2843.181 & 2063.971 & 2892.823 & 2105.551 & 2510.417 & 2202.419 & 1656.576 \\
\hline & 2316.492 & 3638.642 & 2707.755 & 2342.925 & 1888.582 & 2161.253 & 2030.62 & 2619.948 & 2566.976 & 2225.139 \\
\hline & 1166.728 & 2164.912 & 2434.474 & 5222.222 & 1985.586 & 1436.245 & 3797.686 & 2431.133 & 2979.179 & 2265.557 \\
\hline & 1712.398 & 1502.576 & 3261.918 & 2655.5 & 2279.73 & 3340.497 & 1646.382 & 1429.36 & 1858.124 & 1774.41 \\
\hline & 1647.704 & 4916.522 & 1849.932 & 2967.31 & 1728.192 & 1897.477 & 5154.964 & 2144.867 & 1577.087 & 3125.238 \\
\hline & 2439.671 & 2104.491 & 1893.723 & 1874.665 & & & & & & \\
\hline \multirow[t]{4}{*}{ HPVI8 } & 3154.542 & 3641.808 & 1515.848 & 2310.124 & 1093.113 & 1469.67 & 1713.048 & 1855.756 & 4099.734 & 1386.055 \\
\hline & 3559.794 & 2703.672 & 1818.182 & 1609.218 & 2865.984 & 2545.523 & 2373.222 & 3004.519 & 1684.753 & 1888.929 \\
\hline & 2394.459 & 2725.331 & 1991.206 & 2197.088 & 3406.733 & 4591.331 & 3361.065 & 1975.728 & 2260.364 & 2260.364 \\
\hline & 657.1647 & 3002.439 & 2701.686 & 2250.497 & 1934.496 & 2568.334 & 2921.722 & 1880.307 & 1956.511 & 2252.623 \\
\hline \multirow[t]{3}{*}{ HPV4S } & 930.5267 & 2370.254 & 2709.707 & 2534.122 & 2015.069 & 1730.238 & 2297.634 & 3022.334 & 1270.237 & 2815.337 \\
\hline & 2938.45 & 2020.809 & 2767.104 & 5597.865 & 3951.184 & 2357.424 & 1355.54 & 2553.204 & 1707.488 & 4247.096 \\
\hline & 1703.077 & 2556.183 & & & & & & & & \\
\hline HPV 33 & 2432.13 & 2091. 109 & 3120.362 & 2311.529 & 2473.665 & 2158.14 & 2324.836 & 2119.983 & 1408.008 & \\
\hline HPV58 & 1128.311 & 2858.371 & 2366.829 & 2376.098 & 2291.37 & 2014.21 & 1618.629 & & & \\
\hline HPVS2 & 1697.445 & 2044. 625 & 2234.076 & 2139.675 & 3684.375 & 2006.006 & 4102.702 & & & \\
\hline HPV 35 & 2949.153 & 2790.045 & 3892.977 & 1958.556 & 2585.46 & 2104.798 & & & & \\
\hline HPV3। & 2625.671 & 1950.943 & 1299.63 & 2647.585 & 2553.647 & & & & & \\
\hline HPV39 & 1697.842 & 2086.379 & 4097.3 & 1577.537 & 2608.053 & & & & & \\
\hline HPV59 & 1868.574 & 2101.489 & 1554.842 & & & & & & & \\
\hline HPV73 & 2580.611 & 1206.448 & & & & & & & & \\
\hline HPV68 & 1188.425 & 1081.295 & & & & & & & & \\
\hline HPVSI & 3043.692 & & & & & & & & & \\
\hline HPVS6 & 4839.733 & & & & & & & & & \\
\hline \multirow[t]{2}{*}{ HPV. } & 2264.351 & 3321.83 & 3094.797 & 1934.15 & 4000.556 & 2589.056 & 2023.237 & 4510.076 & 3353.836 & 1828.154 \\
\hline & 4086.348 & 2522.704 & 2999.424 & 3108.258 & 4871.563 & 2112.782 & 3276.191 & 4409.535 & 2053.58 & 3526.625 \\
\hline
\end{tabular}

Figure 2. HK2 mRNA expression in different HPV subtype infection from TCGA. The heatmap produced with HK2-normalized counts from 307 cervical cancer specimens in TCGA with data distributed according to HPV infection status. Green is aligned to the largest normalized count which means the highest expression of HK2 and the deepest red is aligned to the smallest normalized count which means the lowest expression of HK2.

nized the HK2 level in 3 normalized counts, which represent the mRNA expression levels of HK2 from 307 cervical cancer specimens from TCGA, into a heatmap according to infection with different HPV subtypes (Fig. 2), we did not observe an obvious connection between HK2 expression and each HPV subtype infection.

The association between HPV16 E7 and HK2 expression. Using 4 different cervical cancer cell lines, we sought to explore the expression levels of certain metabolism-related genes and found that HK2 and Glut1 expression levels were significantly weaker in HPV(-) cell lines, relative to the three HPV(+) cells (Fig. 3). Next, we investigated whether HK2, Glut1 expression correlated with the vital oncoproteins of HPV virus, E6 and E7. Our results indicated that the E7 oncoprotein, but not E6, could enhance HK2 expression in both HPV(+) and HPV(-) cell lines at the RNA level (Fig. 4A) and at the protein level (Fig. 4B). E7 could also enhance Glut1 mRNA expression in SiHa cell line, but the difference was not obvious in $\mathrm{C} 33 \mathrm{~A}$ cell line. In order to explore whether the HPV16 E7 acceleration impact on HK2 involves the HIF-1 $\alpha$ pathway, we knocked down HIF-1 $\alpha$ with shRNA. With the
RT-PCR and western blotting results in the SiHa cell line, we found that knock-down of HIF-1 $\alpha$ induced obvious attenuation of HK2, and HPV16 E7 could rescue HK2 expression when HIF-1 $\alpha$ was knocked down, based on the observation that the HK2 expression level in cells with both HIF-1 $\alpha$ knock-down and HPV16 E7 overexpression was much stronger than that in cells with only HIF-1 $\alpha$ knock-down (Fig. 4B and C). Thus, we suspected that HPV16 E7 could promote the expression of HK2 through a mechanism other than the HIF-1 $\alpha$ pathway.

The impact of HK2 on cervical cancer cells in vitro. To determine the impact of HK2 expression on cervical cancer cells, we attempted to knock-down HK2 in both the HPV(+) $\mathrm{SiHa}$ cell line and the HPV(-) C33A cell line. As shown in Fig. 5, in both normal and hypoxic environments, knock-down of HK2 induced significant overexpression of TFAM, which indicated a reinforcement of mitochondrial function, as well as the downregulation of LDHA, which indicated an ablation of lactification ability. Moreover, knock-down of HK2 also significantly abrogated the expression of MIB, mTOR and MGMT in both normal and hypoxic environments (Fig. 5). Next, SRB analysis was used to explore the impact of $\mathrm{HK} 2$ on proliferation ability of cervical 


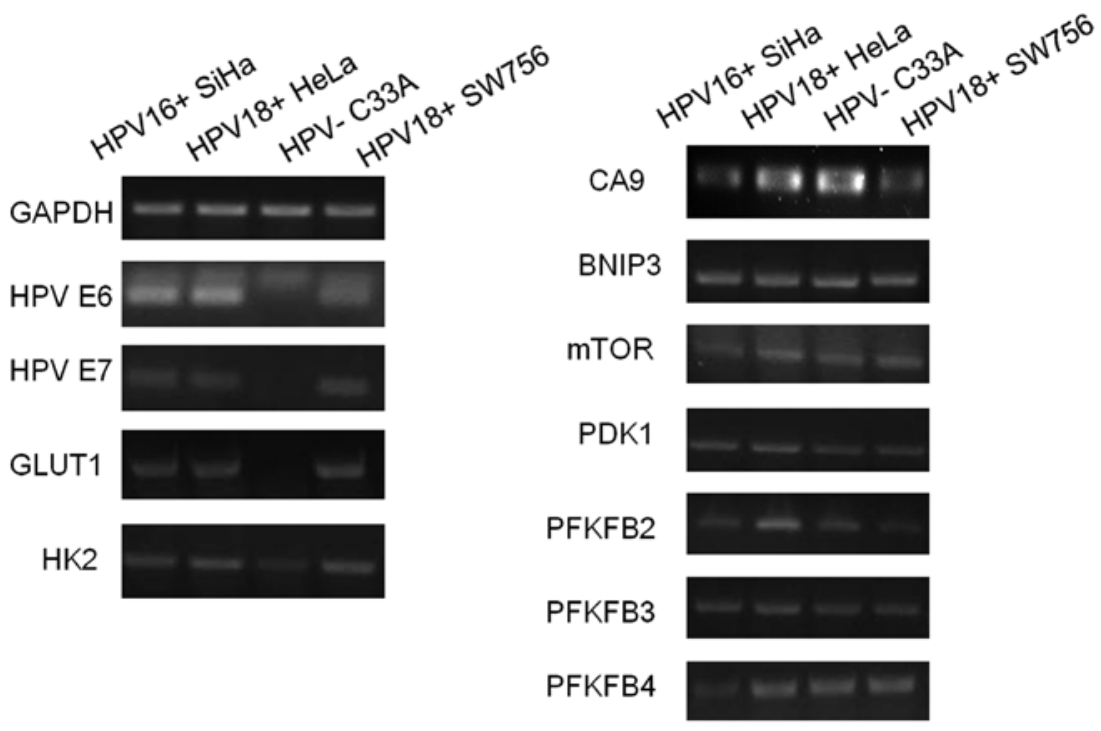

Figure 3. Gene mRNA expression between different cervical cancer cell lines. RT-PCR showing the expression of HK2, Glut1, BNIP3, mTOR, PDK1, PFKFB2, PFKFB3 and PFKFB4 in HPV16(+) SiHa, HPV18(+) HeLa, HPV(-) C33A and HPV18(+) SW756 cell lines.

A

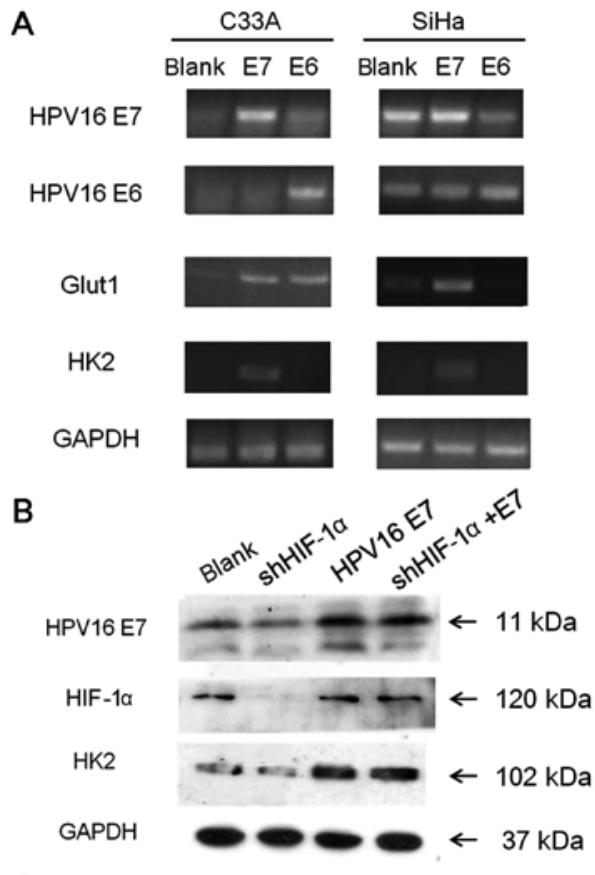

C

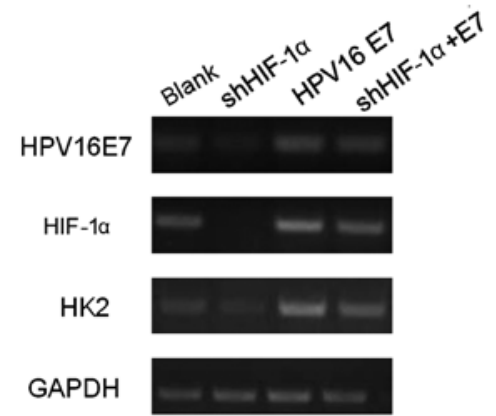

Figure 4. Gene expression change after HPV virus overexpression, HIF-1a or HK2 knock-down. (A) RT-PCR shows elevated expression of HK2 following HPV16 E7 overexpression in both C33A and SiHa cells and elevated expression of Glut1 following HPV16 E7 overexpression in SiHa cells. (B and C) RT-PCR and western blotting show that HPV16 E7 promotes HK2 expression and rescues HK2 expression in both HIF-1 $\alpha$-knock-down and HPV16 E7-overexpressing SiHa cells. The data shown are from a single representative experiment performed in triplicate.
$\mathrm{SiHa}$

C33A

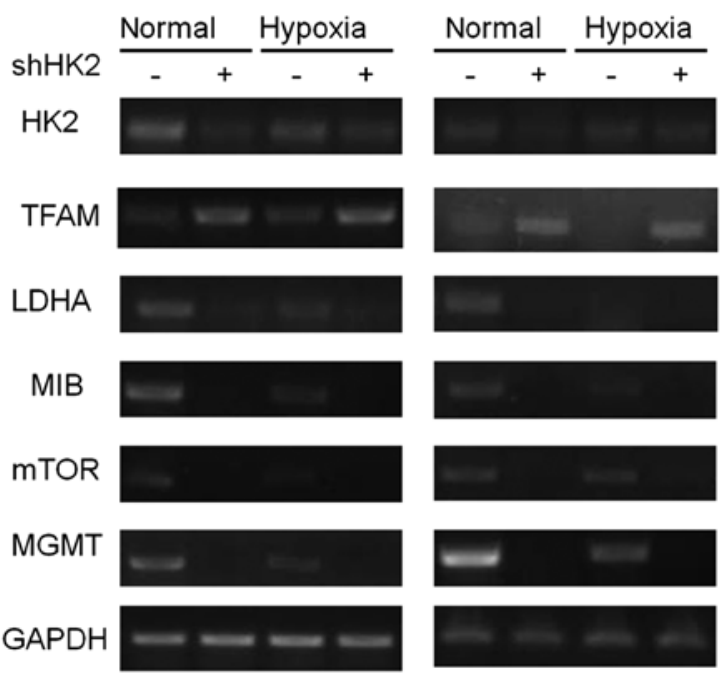

Figure 5. Effect of HK2 knockdown on gene RNA expression in normoxic and hypoxic environments. RT-PCR shows the overexpression of TFAM and downregulated LDHA, MIB, mTOR, and MGMT expression after HK2 knock-down in both normoxic and hypoxic environments in SiHa and C33A cell lines.

cancer cells. After knocking down HK2 expression, cervical cells demonstrated significantly attenuated proliferation ability in normal and hypoxic environments compared to shNC cells (Fig. 6A and B), with the exceptions of SiHa cells in a normal environment and HeLa cells in a hypoxic environment, even though the cervical cancer cells could not grow vigorously after three days of incubation in the hypoxic environment. In order to investigate the influence of HK2 on the radiation sensitivity of cervical cancer cells, we compared their proliferation abilities following irradiation exposure. We found that $10 \mathrm{~Gy}$-irradiated shHK2 SiHa and C33A cells exhibited much greater proliferation-inhibiting effects compared to the 10 Gy-irradiated shNC group, even though cells did not grow vigorously three days after irradiation (Fig. 6C). Next, we treated the cervical 
A

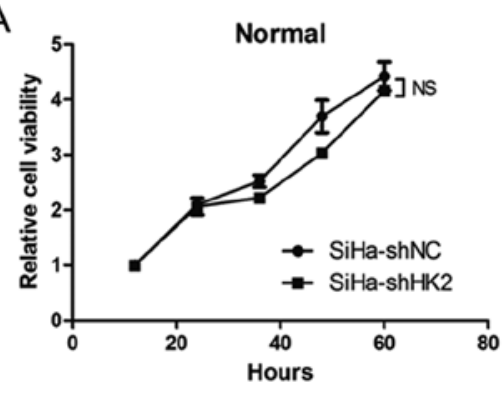

B
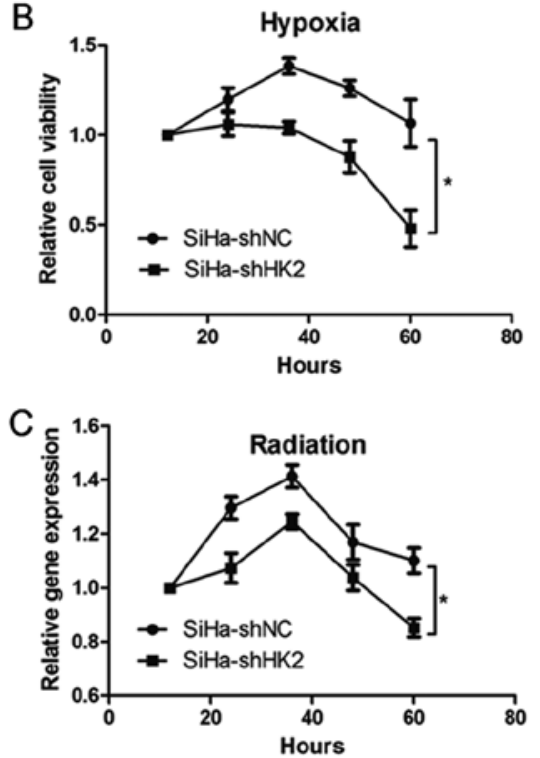

D

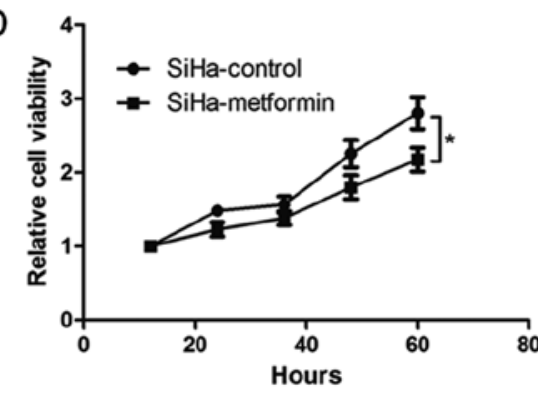

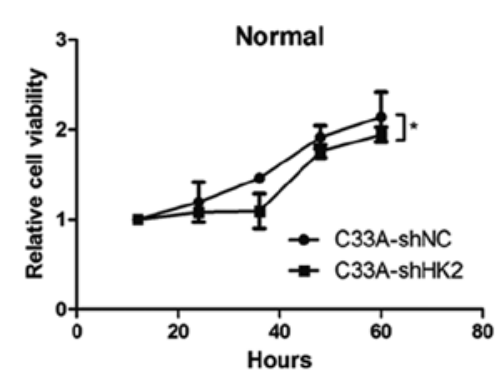
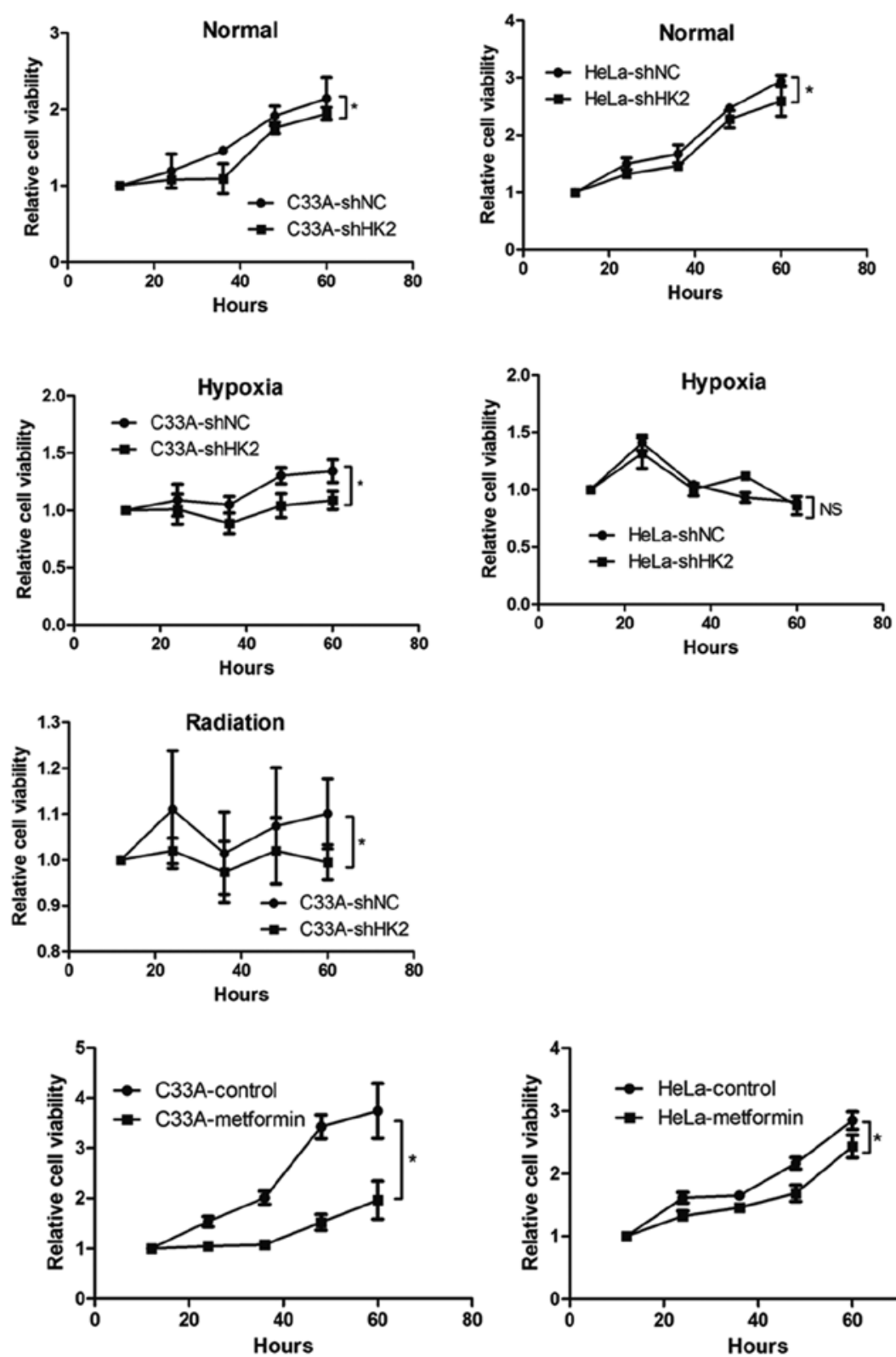

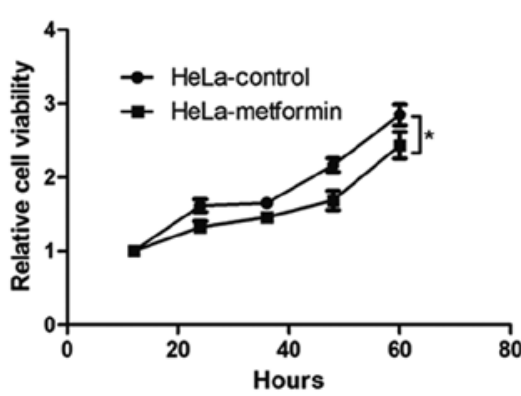

Figure 6. Effect of HK2 knockdown and metformin treatment on cell proliferation in different environments. (A) shHK2-containing C33A and HeLa cell lines demonstrating decreased proliferation compared to the shNC-containing cells in the normoxic environment. (B) shHK2 SiHa and C33A cell lines demonstrate decreased proliferation relative to shNC cells in a hypoxic environment. (C) shHK2 SiHa and C33A cell lines demonstrate decreased proliferation versus the shNC cells after 10-Gy irradiation. (D) Metformin-treated groups of SiHa, C33A and HeLa cells demonstrate decreased proliferation relative to the control groups. Each data point represents the mean of triplicates $(\mathrm{N}=3)$ with standard error of the mean $(\mathrm{SEM})$ indicated by error bars. * $\mathrm{p}<0.05$.

cancer cells with metformin, which is reported to inhibit HK2 function (35-38), and has been reported to impair glucose metabolism and tumor growth in breast cancer (39). We found that the proliferation of the metformin-exposed group was significantly reduced compared to the control group (Fig. 6D). Therefore, these results indicate that HK2 may exert a variety of impacts on cervical cancer cells, including cell metabolism, the mTOR pathway and DNA damage. Moreover, HK2 inhibition specifically attenuated the proliferation of cervical cancer cells.

The effect of HK2 on cervical cancer cell apoptosis in vitro. Next, the impact of HK2 on apoptosis in cervical cancer cell lines in normal and hypoxic environments and after radia- tion exposure was explored. Cell survival was assessed using Annexin V-PE and 7AAD staining. In SiHa cells, the shHK2 group demonstrated an increase in apoptosis, particularly in later apoptotic events, compared to shNC control cells in normal and hypoxic environments as well as after 10-Gy of irradiation (Fig. 7A-C). In C33A cells, the shHK2 group displayed an increase in apoptosis compared to shNC control cells in earlier apoptosis in normal condition (Fig. 7A), and also in both earlier and later apoptosis in the hypoxic environment and after 10 Gy of irradiation (Fig. 7B and C). Similarly, in $\mathrm{SiHa}, \mathrm{C} 33 \mathrm{~A}$ and HeLa cells, metformin led to an increase in apoptosis compared to control group cells, especially in the C33A and HeLa cell lines (Fig. 7D). To further explore the molecular mechanisms underlying the anti-apoptotic role of 
A
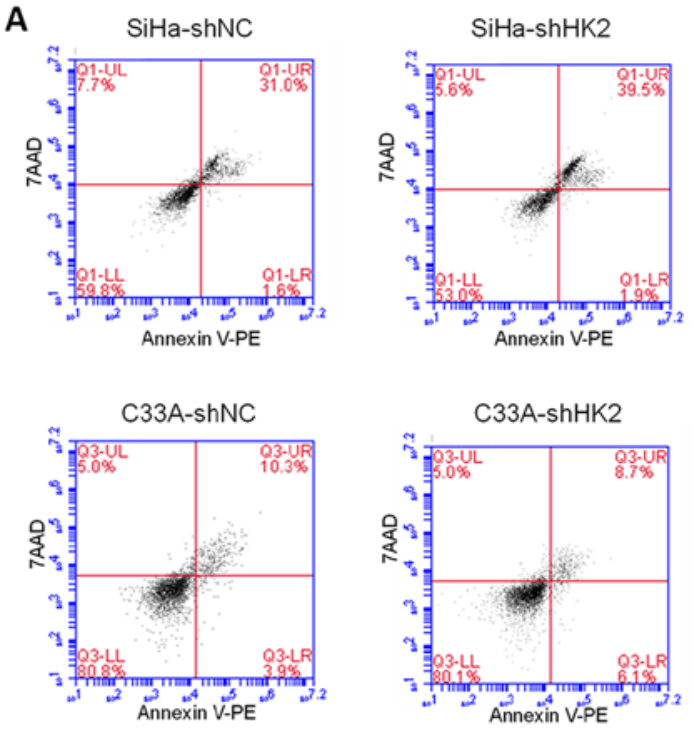

C
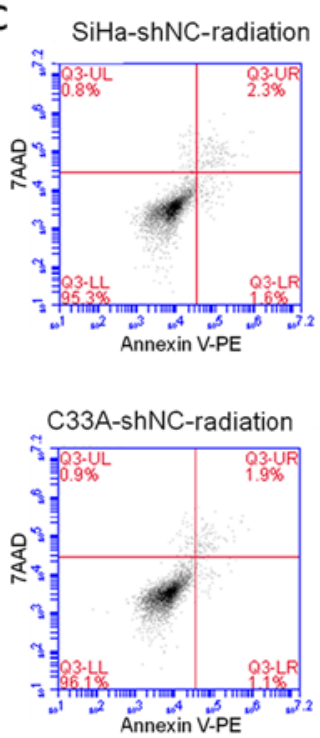

B
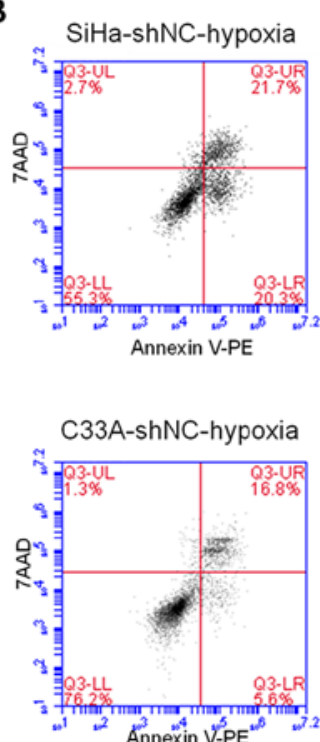

C33A-shHK2-hypoxia

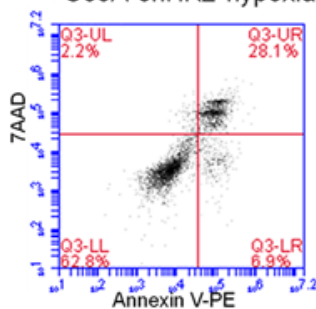

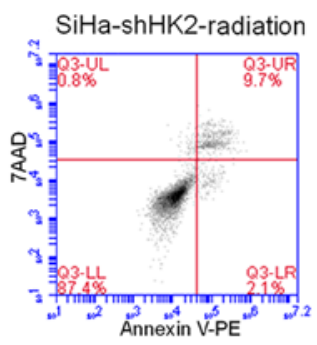

D
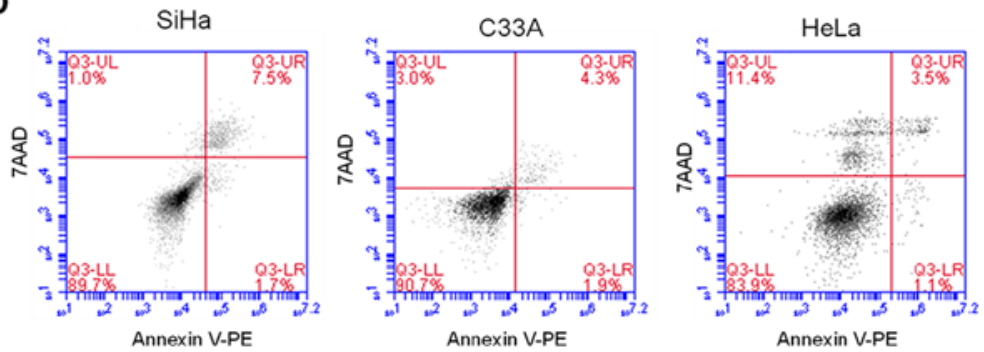

C33A-shHK2-radiation
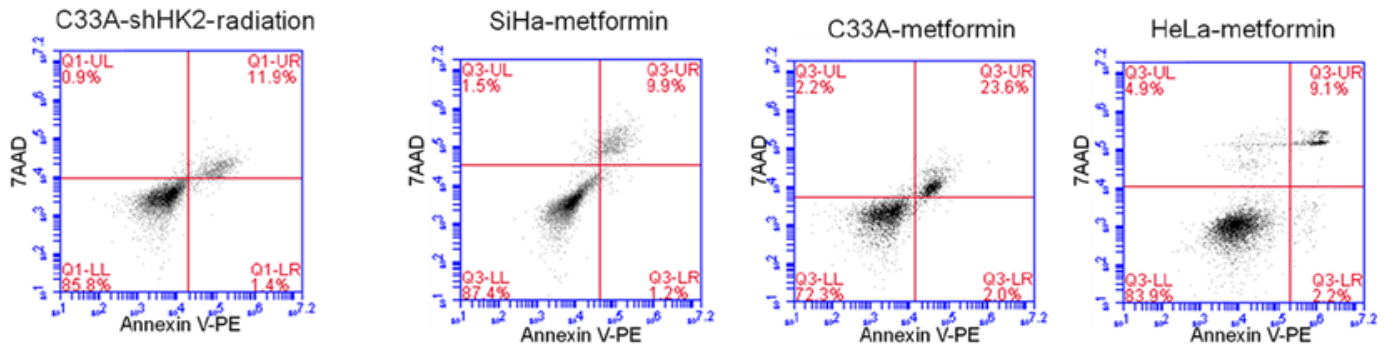

Figure 7. Effects of HK2 knockdown or metformin treatment on cell apoptosis in different environments. ShHK2 SiHa cell line exhibits a greater degree of apoptosis, especially in later apoptotic events, compared to the shNC cells in a normoxic environment (A), in a hypoxic environment (B), and after 10 Gy of irradiation (C). ShHK2 C33A cell line displays an increase in apoptosis compared to the shNC in earlier apoptosis in the normoxic condition (A), and in both earlier and later apoptosis in the hypoxic environment (B) and after $10 \mathrm{~Gy}$ of irradiation (C). Metformin-treated SiHa, C33A and HeLa cells exhibit a greater degree of apoptosis compared to the control group (D). The data shown are from a single representative experiment performed in triplicate.

HK2, we examined two biochemical markers of apoptosis, polyadenosine diphosphate ribose polymerase (PARP) and cleaved caspase-3, in the shHK2 and shNC stable cell lines. Cleaved PARP and caspase- 3 were detected at higher levels in the shHK2 cell lines than in the shNC cell lines in normal environment (Fig. 8A). We also found higher expression of cleaved PARP and cleaved caspase- 3 in shHK2 cell lines than shNC cell lines in the hypoxic environment and after irradiation, respectively (Fig. 8B and C). The B-cell lymphoma (Bcl) family of proteins is known to be closely associated with apoptosis. As shown in Fig. 8A, a significant decrease in the anti-apoptotic protein Bcl-2 was observed in shHK2 cells relative to shNC cells. Taken together, inhibition of HK2 promoted the apoptotic potential of cervical cancer cells.
Metabolic changes in cervical cancer cells. Since HK2 serves as the most critical enzyme regulating glycolysis, we investigated the metabolic profile of cervical cancer cells using the XF analyzer. In all three shNC cell lines, the ECAR was nearly two times higher than that of the respective shHK2 cell line under glucose starvation conditions, indicating that HK2 knock-down resulted in a strong inhibition of glycolysis in all cell lines tested (Fig. 9A-C). Glycolytic ECAR was measured immediately after the injection of glucose. Glucose addition usually stimulated glycolysis, but there was a noticeable inhibition of glycolysis in the shHK2 group compared to shNC group, indicating that the shHK2 group completely lacked glycolytic flexibility upon glucose exposure (Fig. 9). By treating the cells with oligomycin, which reduces mitochon- 
A
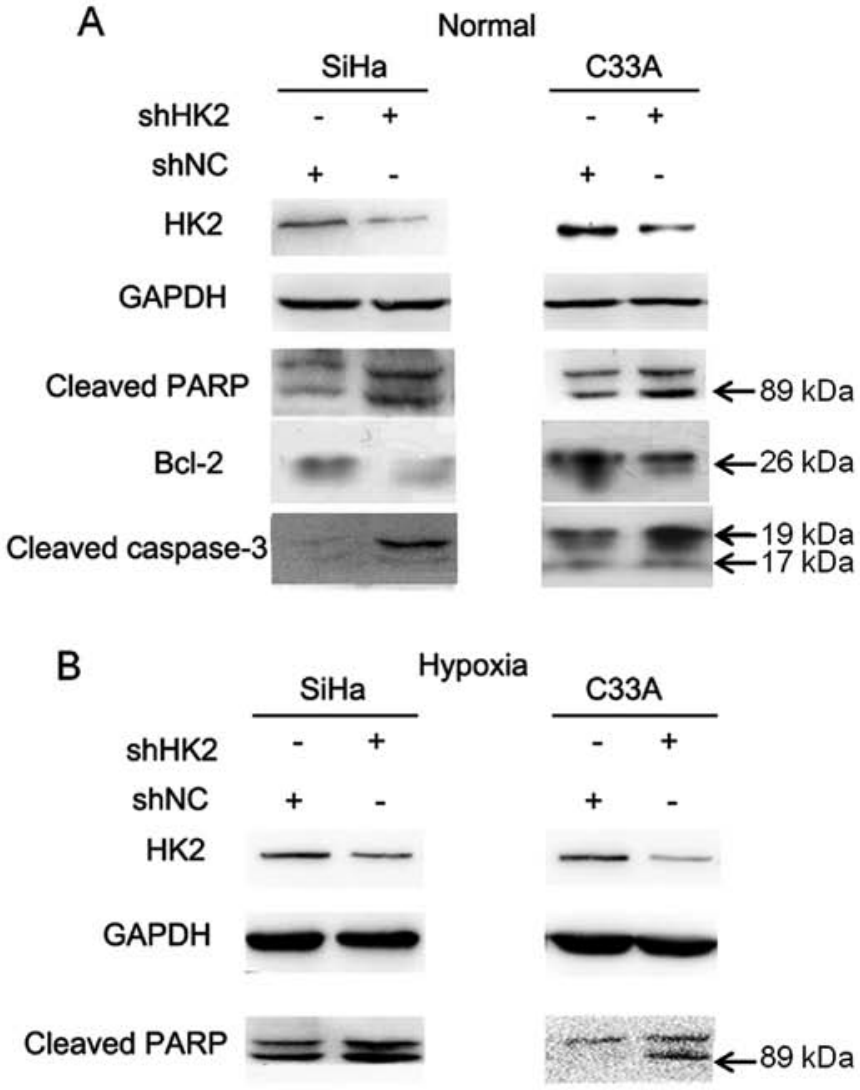

C

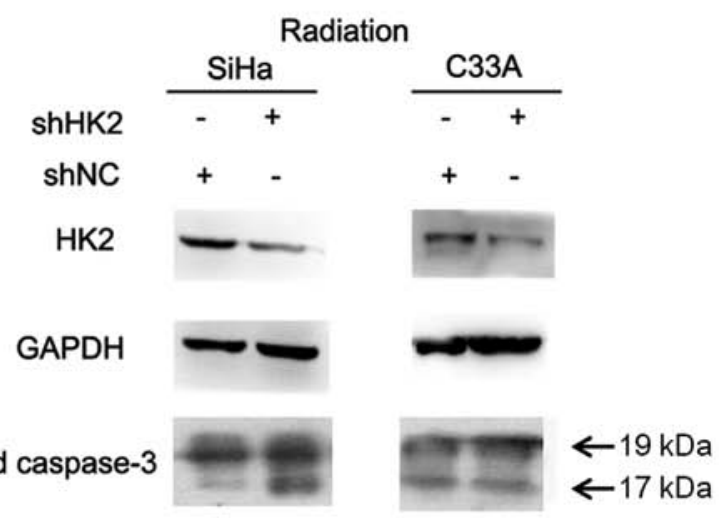

Figure 8. Effects of HK2 knockdown on apoptosis-related gene expression in different environments. (A) Western blot analysis demonstrates increased levels of cleaved caspase-3 and cleaved PARP and lower expression of Bcl-2 in the shHK2 SiHa and C33A cell lines compared to shNC groups in a normoxic environment. (B) Western blot analysis demonstrates increased levels of cleaved PARP in the shHK2 SiHa and C33A cell lines compared to shNC groups in a hypoxic environment. (C) Western blot analysis demonstrates increased levels of cleaved caspase- 3 in the shHK2 group of $\mathrm{SiHa}$ and $\mathrm{C} 33 \mathrm{~A}$ cell lines compared to shNC groups after irradiation. The data shown are from a single representative experiment performed in triplicate.

drial respiration and maximizes glycolytic ATP production, we calculated the complete cellular glycolytic capacity. We observed more reserved glycolytic capacity in HeLa-shNC than in HeLa-shHK2 cells (Fig. 9C). The addition of 2-deoxyglucose (2-DG), an inhibitor of glycolysis, was intended to ensure that the ECAR measured was a result of glycolytic metabolism, and we confirmed that the ECAR returned to non-glycolytic levels in both shNC and shHK2 HeLa cell lines after 2-DG treatment (Fig. 9C). Basal measurements of the mitochondrial respiration rate (OCR) and glycolytic ECAR were measured to study the change in metabolic profiles between shNC and shHK2 cervical cancer cells. Intriguingly, quantitation showed reduced ECAR and increased OCR rates upon HK2 knock-down in HeLa cells (Fig. 10B), suggesting a partial metabolic profile switch to oxidative phosphorylation in HeLa-shHK2 cells. The SiHa and C33A cells showed both reduced ECAR and OCR rates upon HK2 knock-down, which demonstrated a decreased metabolic rate (Fig. 10A and C). Additionally, metformin treatment of $\mathrm{SiHa}$ cells revealed a higher OCR/ECAR ratio, indicating a partial metabolic alteration to oxidative phosphorylation in SiHa cells when HK2 function was inhibited (Fig. 10D). Finally, SiHa cells transfected with HPV16 E7 plasmids displayed a high ECAR/OCR ratio, demonstrating a partial metabolic switch to glycolysis when HPV16 E7 was overexpressed (Fig. 10E).

HK2 knock-down xenograft model. Finally, we performed a xenograft tumor growth assay in nude mice to further evaluate the tumorigenic role of HK2 in cervical cancer cells. First, mice were subcutaneously injected with shRNA or shNC SiHa cells. After five weeks, the average size of the tumors in the shHK2 group was substantially smaller than that in the shNC group (Fig. 11A-C, $\mathrm{p}<0.05$ ). To determine the effect of HK2 on apoptosis, we performed IHC of caspase-3 and PARP in the sectioned tumor tissues. Considerably stronger caspase-3 and PARP staining intensities were detected in shHK2 tumor tissues relative to shNC tissues (Fig. 11D).

\section{Discussion}

A novel hypothesis was developed by us that the glycolytic enzyme HK2 serves as a critical step in aerobic glycolysis inducing irradiation resistance in cervical cancer, offering a proliferative and cell survival advantage. There are some reports demonstrating that HK2 is aberrantly expressed in gynecological cancers, including cervical and ovarian cancer $(40,41)$. For example, Huang et al suggested that HK2, which is located in the cytoplasm of cervical carcinoma cells, shows higher expression levels in a radiation-resistant group than a radiation-sensitive group (42), suggesting common roles for $\mathrm{HK} 2$ as an oncoprotein and an indicator for radiation resistance in gynecology tumors. In our study, we provided evidence demonstrating that downregulation of HK2 restored apoptosis of cervical cancer cells. Moreover, the levels of cleaved caspase- 3 and cleaved PARP in C33A and SiHa cells were significantly accelerated and $\mathrm{Bcl}-2$ expression was inhibited by HK2 inhibition, suggesting an essential role for HK2 in the anti-apoptotic mechanism of cervical cancer cells. The targeted inhibition of HK2 expression by shRNA demonstrated a suppression of tumor growth both in vitro and in vivo. HK2 expression inhibition also attenuated the expression of mTOR, suggesting that HK2 might modulate the PI3K/AKT/mTOR pathway that is a crucial constituent of an adaptive system for sensing the availability of a wide range of growth factors and nutrients in homeostasis (43). Moreover, we found that suppression of HK2 not only inhibited the expression of MIB, it also inhibited the expression of MGMT. Our study demonstrated that HK2 inhibition downregulated distinct pathway proteins including mTOR, MIB and MGMT, that HK2 could serve as 
A

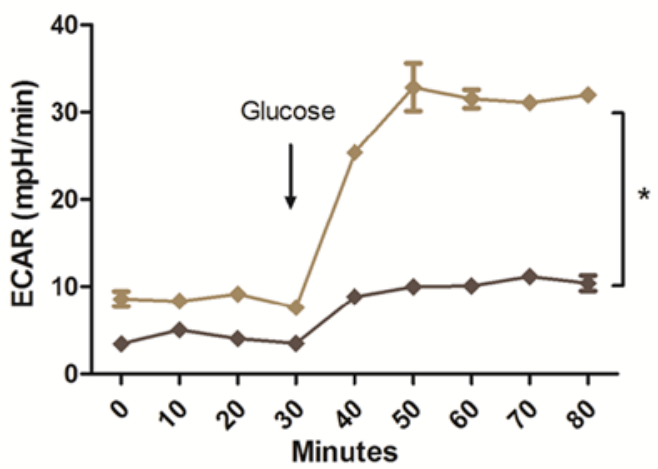

Background $\rightarrow$ SiHa-ShNC $\leadsto$ SiHa-shHK2
B

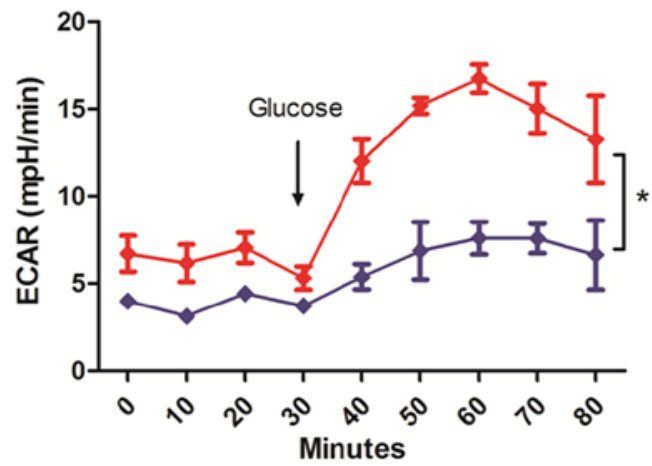

- Background $\rightarrow$ C33A-ShNC $\rightarrow$ C33A-shHK2

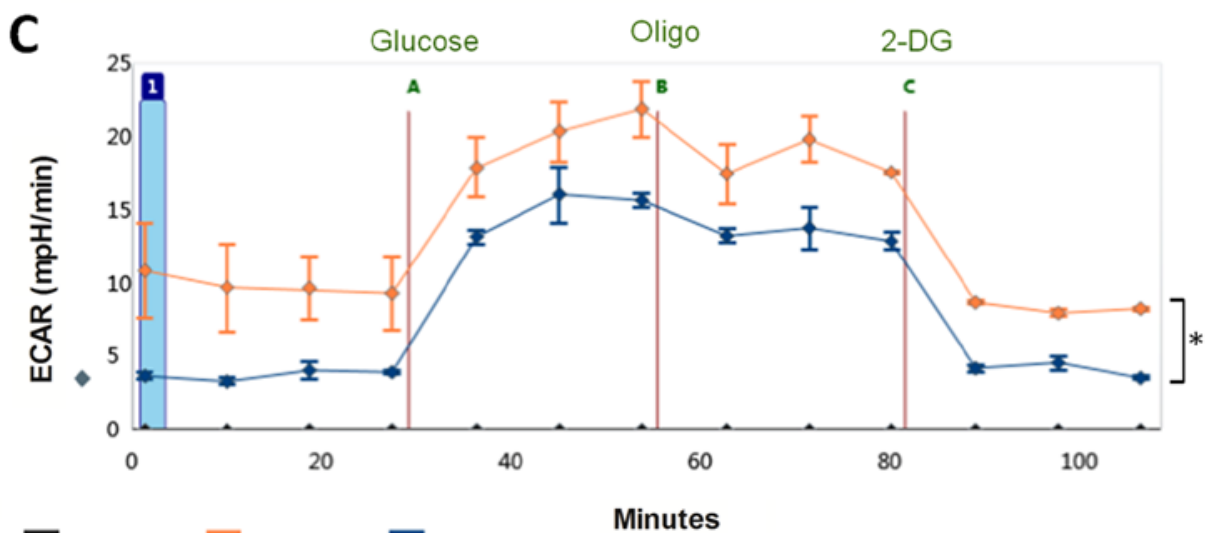

Background HELA-SHNC $\square$ HELA-SHHK2

Figure 9. Real-time ECAR evaluation of glycolysis. (A and B) Knockdown of HK2 in C33A and SiHa cells results in decreased glycolysis and no increase in glycolysis after glucose exposure compared to shNC group. (C) Knockdown of HK2 in HeLa cells results in decreased glycolysis and no increase in glycolysis after glucose exposure, and lower reserved glycolytic capacity compared to shNC group. Each data point represents the mean of triplicates \pm SEM. ${ }^{*}<0.05$.

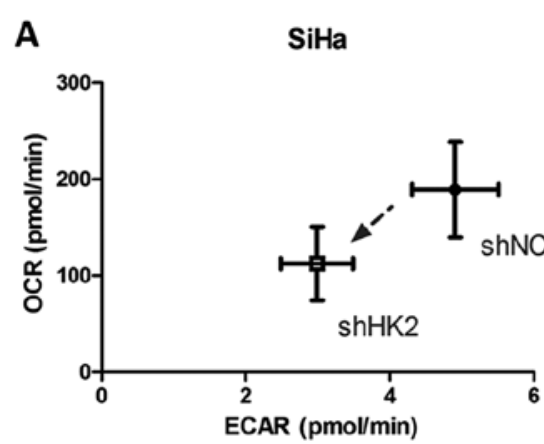

D
B

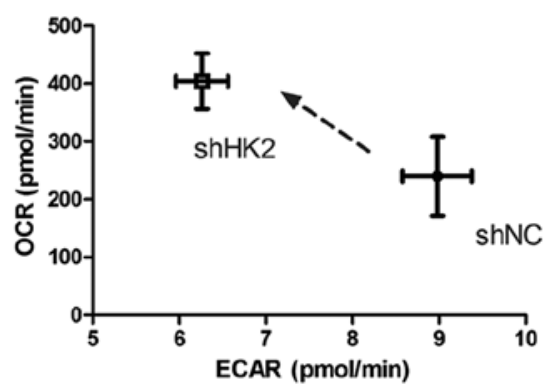

$\mathrm{SiHa}$

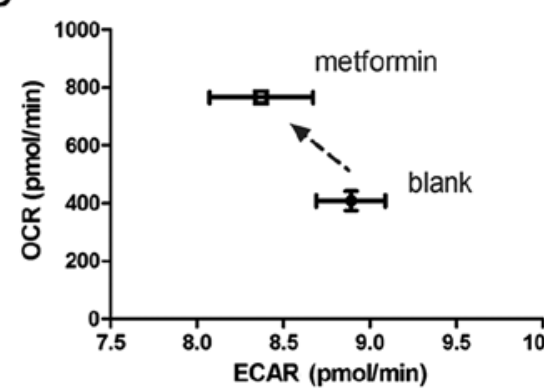

E
C

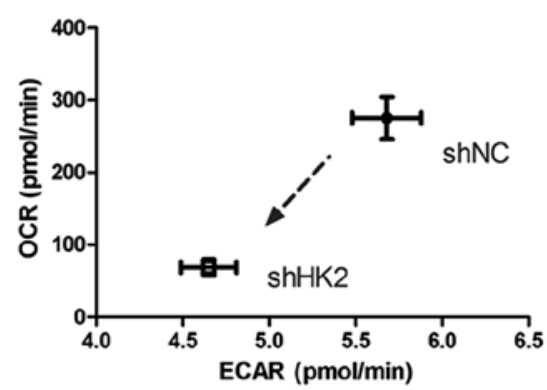

$\mathrm{SiHa}$

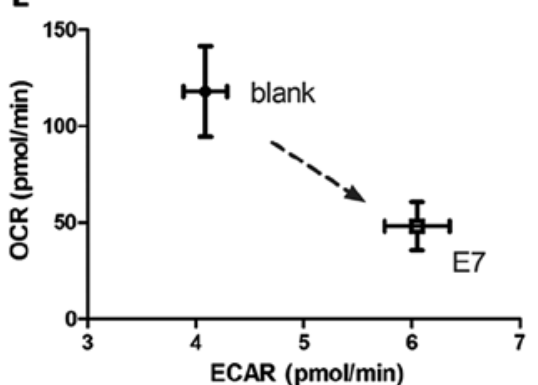

Figure 10. Effects of HK2 knockdown or metformin treatment on OCR and ECAR. (A and C) Knockdown of HK2 in C33A and $\mathrm{SiHa}$ cells reduces $\mathrm{O}_{2}$ consumption (OCR) and extracellular acidification (ECAR) compared to shNC cells. (B) Knockdown of HK2 HeLa cells reduces OCR and increased ECAR compared to shNC cells. (D) Metformin treatment of SiHa cells induced increased OCR and reduced ECAR. (E) HPV16 E7-overexpressing SiHa cells display decreased OCR and increased ECAR compared to the blank group. The changes of ECAR and OCR are statistically significant between different groups $(\mathrm{p}<0.05)$ and the bars represent SEM. 

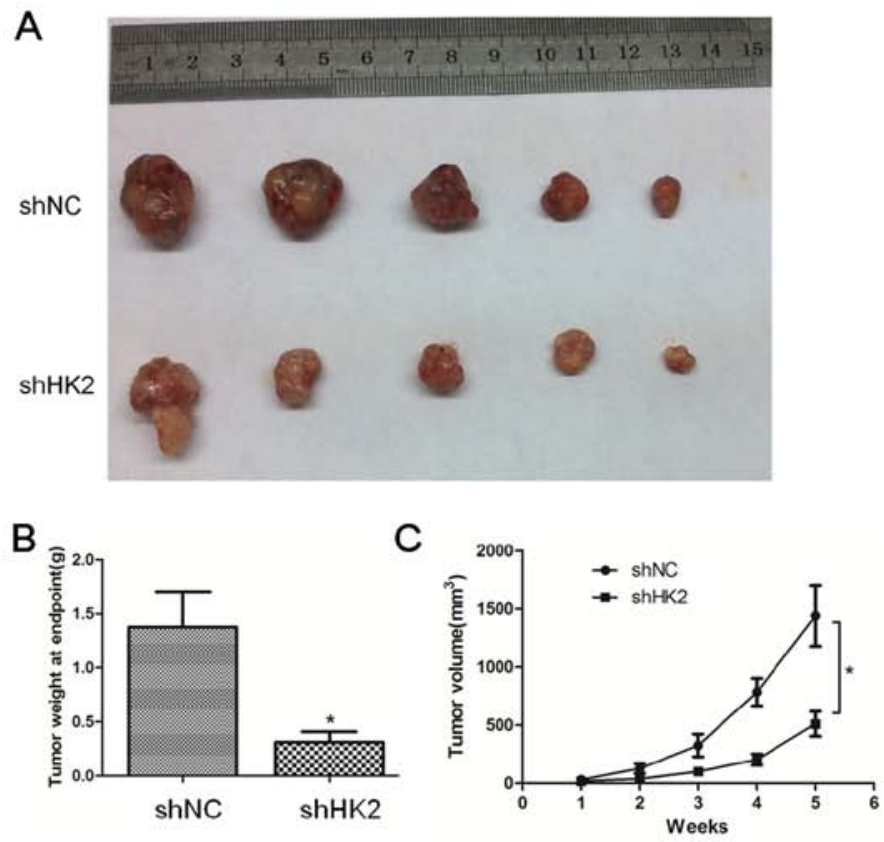

D

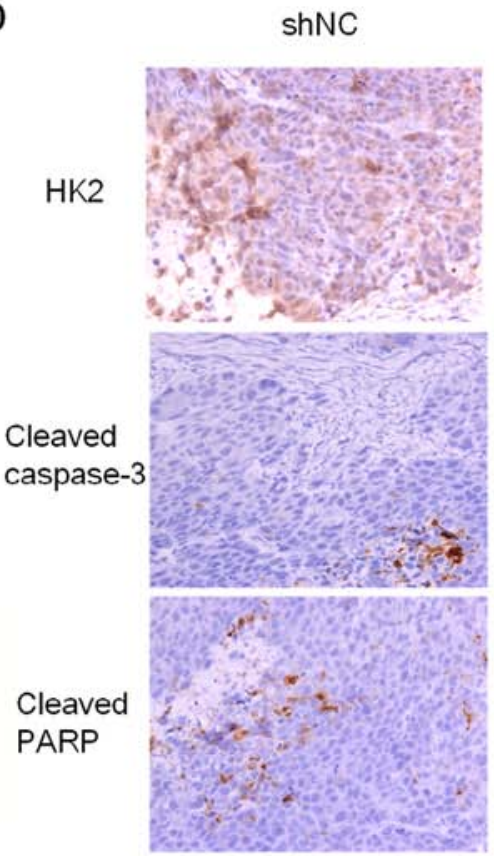

shHK2

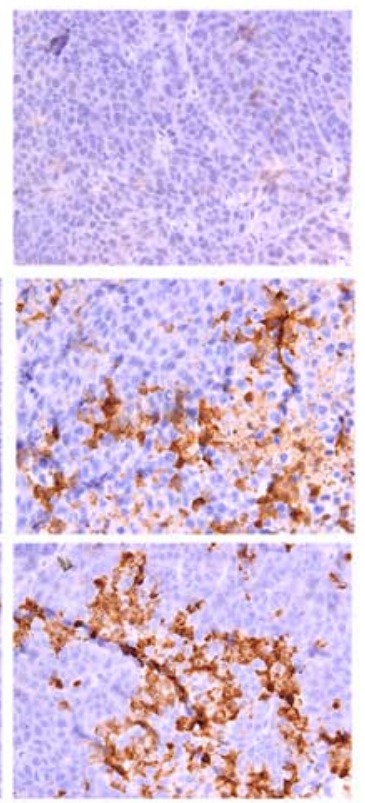

Figure 11. Effects of HK2 knockdown on tumorigenicity in a nude mouse xenograft model. (A) Tumors of mice injected with shNC- or shHK2-transfected SiHa cells and removed five weeks after injection. (B) Weights of shNC and shHK2 tumors at endpoint. Values are the means \pm SD of five tumors in each group. ${ }^{*} \mathrm{p}<0.05$. (C) Volumes of each tumor were measured each week for five weeks. Values are the means \pm SD of five tumors in each group. ${ }^{*}<<0.05$. (D) Immunohistochemical staining of HK2, cleaved caspase-3, and cleaved PARP in the shNC and shHK2 xenograft tumors.

a biomarker and potential therapeutic target of cervical cancer treatment.

In order to promote the effect of radiation therapy to cervical cancer patients, it is most crucial to understand the mechanism of radiation resistance. Hypoxia, as a common microenvironment for malignant cells, is the fundamental reason (22). HIF-1 $\alpha$, which is stabilized upon hypoxia, helps the radiation resistance (23) and can encourage a variety of functional changes including tumorigenesis and metastasis and the glycolytic process $(44,45)$. Glycolysis contributes to the radioresistance for the following reasons (46-48). Firstly, the accumulation of glycolytic products builds a redox buffer network which removes free radicals and ROS produced by ionizing radiation from irradiation therapy. The effect of irradiation therapy could be significantly attenuated by the rescuing buffer network from aerobic glycolysis in a hypoxic environment $(49,50)$. Secondly, glycolysis not only produces the anabolic precursors for de novo synthesis of nucleotides and lipids, which are necessary for high tumor growth rate, but also supplies the tumor cells with plenty of ATP in a hypoxic microenvironment as a vital energy contributor and facilitate DNA repair in cells $(26,27,51)$. Thus, if we could attenuate cancer cell glycolysis, the rescue network and energy contributor for cancer cell survival would be greatly alleviated and radiasensitization would be intensified. Unfortunately, there is limited clinical research designed to attenuate radio-resistance through modifying hypoxia and glycolysis. Thereby, we aimed to elicit a novel way to target the critical enzyme of glycolysis, suppress tumor glycolysis to enhance the ionizing radiation from irradiation therapy, block the main energy supply, and ultimately increase the sensitivity of cervical cancer cells to radiation therapy. HK2, being the first irreversible critical modulator of glycolysis, is on the top of the list of genes poten- tially modified and regulated. Although some HK inhibitors are already in phase I and II clinical trials $(52,53)$, there are still restrictions involved in the wide range of regulatory pathways of HK inhibitors, warranting further research.

In the present study, we focused on HK2 inhibition to switch cervical cancer cell metabolism to one less dependent on glycolysis, aiming to reduce the impact of glycolysis on ionizing radiation, block the main energy contributor, induce cancer cells apoptosis and eventually improve cervical cancer sensitivity to radiation therapy. We observed that HK2 inhibition with shRNA or metformin could effectively suppress ECAR and glycolytic metabolism in cervical cancer cell lines with dinimishing expression of LDHA and simultaneously accelerate the OCR and enhance oxidative phosphorylation with accelerating expression of TFAM. We indicated that HK2 inhibition managed to impair cervical cancer cell lactification ability and reinforce mitochondrial function. At the same time, shNC-containing cervical cancer cell lines exhibited superior proliferation abilities comparable to the HK2 knock-down cell lines in both normal and hypoxic environments as well as after radiation exposure. Irradiated cervical cancer cells displayed significantly inferior proliferation after HK2 inhibition. Due to the protecting shield for cancer cells created by glycolysis in hypoxia, the anabolic precursors for tumor growth and plenty of the ATP produced by glycolysis were severely blocked by HK2 inhibition, HK2 knockdown cells tend to lose some of the survival chance under irradiation circumstance and showed more sensitivity to irradiation than shNC group. Similarly, others reported that systemic deletion of HK2 is therapeutic (7), and from the data displayed above, we proposed that inhibition of HK2 could prevent the glycolysis of cancer cell, suppress proliferation of cervical cancer cells, enhance apoptosis and most importantly intensify the sensitivity of cervical cancer 
cells to radiation therapy. Metformin directly inhibits HK2 activity and subcellular localisation inducing dissociation of HK2 from the mitochondria. Metformin impairs glycolysis and has an inhibitory effect on AKT phosphorylation which contributes to effects on HK2 suppression by decreasing HK2 expression, activity and mitochondrial interaction $(35-38,45)$. We showed that metformin served as an HK2 inhibitor, contributed to the apoptosis of cervical cancer cells, suppressed the proliferation and altered the metabolic profile of cervical cells to less dependent on glycolysis.

Human papilloma virus (HPV) is a small, circular, doublestranded DNA virus infecting epithelial cells and has been reported to be necessary, but not sufficient to initiate cervical squamous epithelial cell tumorigenesis $(54,55)$. HPV E7, as the vital oncoprotein of this virus, plays an important role in the viral life cycle by impacting the tight link between cellular proliferation and differentiation in normal epithelium, thus leading the virus to replicate in differentiating epithelial cells (56). In our study, we first identified HK2 expression in different cervical cancer cell lines. Obvious expression of HK2 was detected only in HPV(+) cervical cancer cell line but not in the HPV(-) cell line. We elucidated a close relationship between the HPV16 E7 oncogene and HK2 expression. It has been reported that the HPV E7 protein enhances HIF-1 $\alpha$ transcriptional activity through manipulating the response to hypoxia (57), and displacing the histone deacetylases HDAC1, HDAC4, and HDAC7 $(57,58)$. Both HPV E6 and E7 are independently capable of inducing expression of HIF-1 $\alpha$ upon DFO treatment $(59,60)$. It has also been shown that the radioresistance-associated HIF1 protein upregulates many enzymes of the glycolytic process, including HK2, through binding to the hypoxia responsive elements (HREs) of the promoter $(5,61,62)$. What we discovered was HPV16 E7 was directly responsible for the up-modulation of $\mathrm{HK} 2$ by a pathway independent of HIF-1 $\alpha$. There is a common mechanism that HK2 expression is impacted by transcription factors in tumor cells (63). The key oncogenic pathways present in multiple cancers, such as PI3K/Akt signaling, enhance the expression of the glycolytic enzyme HK2, which further hinders cell apoptosis, facilitating tumor growth and progression (64). In our study, we provide the first evidence that the HPV oncoprotein E7 as one of those transcriptional factors, could exert an enhancing impact on HK2 expression independent of the HIF-1 $\alpha$ pathway. On the other hand, we found that HPV16 E7 overexpression could effectively make SiHa cells more dependent on the glycolytic metabolic profile through increasing ECAR and reducing OCR, facilitating the Warburg effect in tumor cells, and knockdown of HK2 or metformin treatment significantly abrogated glycolysis by reducing ECAR. Thus, we postulated that HPV16 E7 increases glycolytic metabolism and promotes HK2 expression and its regulation on downstream glycolysis metabolism. This suggests an underlying mechanism through which HPV E7 induces the Warburg effect via pathways including enhancing the expression or functions of diverse glycolytic enzymes, namely HK2. We propose an essential role for the HPV16 E7 oncogene in the Warburg effect through regulation of the critical rate-limiting enzyme of glycolysis, HK2. Therefore, if we could effectively inhibit HK2 expression or function, we could eventually abrogate the HPV16 E7-induced glycolytic metabolism phenotype, blocking the main energy sources of cancer cells, suppress tumor growth and progression and enhance the sensitivity of $\mathrm{HPV}(+)$ cervical cancer cells to irradiation therapy.

In conclusion, we have successfully identified an essential role for HK2 in the HPV16 E7-induced glycolytic metabolic profile. We further demonstrated that HK2 inhibition not only suppress cervical cancer cell energy metabolism, which is a hypoxia-facilitated glycolytic process, and sensitive HPV16 E7-induced cervical cancer cells to irradiation, it also suppresses cervical cancer cell proliferation, survival and carcinogenesis, both in vivo and in vitro. Furthermore, HPV16 E7 increases glycolytic metabolism and promotes HK2 expression and its regulation on downstream metabolism. Our data extend the understanding of the regulatory network of HK2 in cervical cancer metabolism and indicate potential targets for the exploitation of cervical cancer irradiation therapy strategies.

\section{Acknowledgements}

We thank Dr Sufang Wu for kindly providing pCAG-mycHPV16 E7, pCAG-myc-HPV16 E6 and pCAG-myc-blank plasmids. This study was supported by grants from the National Natural Science Foundation of China (NSFC nos. 81172476, 811272885 and 81472427), Shanghai Science and Technology Committee Foundation Basic Research Field focused Project (13JC1404501), Doctoral Specialized Fund from Ministry of Education (20120073110090).

\section{References}

1. Chen W, Zheng R, Baade PD, Zhang S, Zeng H, Bray F, Jemal A, Yu XQ and He J: Cancer statistics in China, 2015. CA Cancer J Clin 66: 115-132, 2016.

2. Kroemer G and Pouyssegur J: Tumor cell metabolism: Cancer's Achilles' heel. Cancer Cell 13: 472-482, 2008.

3. Hsu PP and Sabatini DM: Cancer cell metabolism: Warburg and beyond. Cell 134: 703-707, 2008.

4. Mathupala SP, Ko YH and Pedersen PL: Hexokinase II: Cancer's double-edged sword acting as both facilitator and gatekeeper of malignancy when bound to mitochondria. Oncogene 25: 4777-4786, 2006.

5. Mathupala SP, Rempel A and Pedersen PL: Glucose catabolism in cancer cells: Identification and characterization of a marked activation response of the type II hexokinase gene to hypoxic conditions. J Biol Chem 276: 43407-43412, 2001.

6. Wang W, Liu Z, Zhao L, Sun J, He Q, Yan W, Lu Z and Wang A: Hexokinase 2 enhances the metastatic potential of tongue squamous cell carcinoma via the SOD2 $-\mathrm{H}_{2} \mathrm{O}_{2}$ pathway. Oncotarget 8: 3344-3354, 2017.

7. Patra KC, Wang Q, Bhaskar PT, Miller L, Wang Z, Wheaton W, Chandel N, Laakso M, Muller WJ, Allen EL, et al: Hexokinase 2 is required for tumor initiation and maintenance and its systemic deletion is therapeutic in mouse models of cancer. Cancer Cell 24: 213-228, 2013.

8. Wolf A, Agnihotri S, Micallef J, Mukherjee J, Sabha N, Cairns R, Hawkins C and Guha A: Hexokinase 2 is a key mediator of aerobic glycolysis and promotes tumor growth in human glioblastoma multiforme. J Exp Med 208: 313-326, 2011.

9. Bustamante E and Pedersen PL: High aerobic glycolysis of rat hepatoma cells in culture: Role of mitochondrial hexokinase. Proc Natl Acad Sci USA 74: 3735-3739, 1977.

10. Kim JW and Dang CV: Multifaceted roles of glycolytic enzymes. Trends Biochem Sci 30: 142-150, 2005.

11. Mathupala SP, Ko YH and Pedersen PL: Hexokinase-2 bound to mitochondria: Cancer's stygian link to the 'Warburg Effect' and a pivotal target for effective therapy. Semin Cancer Biol 19: $17-24,2009$. 
12. Pastorino JG, Shulga N and Hoek JB: Mitochondrial binding of hexokinase II inhibits Bax-induced cytochrome $c$ release and apoptosis. J Biol Chem 277: 7610-7618, 2002.

13. Majewski N, Nogueira V, Bhaskar P, Coy PE, Skeen JE, Gottlob K, Chandel NS, Thompson CB, Robey RB and Hay N: Hexokinasemitochondria interaction mediated by Akt is required to inhibit apoptosis in the presence or absence of Bax and Bak. Mol Cell 16: 819-830, 2004.

14. Gottlob K, Majewski N, Kennedy S, Kandel E, Robey RB and Hay N: Inhibition of early apoptotic events by Akt/PKB is dependent on the first committed step of glycolysis and mitochondrial hexokinase. Genes Dev 15: 1406-1418, 2001.

15. Rockwell S, Dobrucki IT, Kim EY, Marrison ST and Vu VT: Hypoxia and radiation therapy: Past history, ongoing research, and future promise. Curr Mol Med 9: 442-458, 2009.

16. Shimura T, Kakuda S, Ochiai Y, Nakagawa H, Kuwahara Y, Takai Y, Kobayashi J, Komatsu K and Fukumoto M: Acquired radioresistance of human tumor cells by DNA-PK/AKT/ GSK3beta-mediated cyclin D1 overexpression. Oncogene 29: 4826-4837, 2010.

17. Bolderson E, Richard DJ, Zhou BB and Khanna KK: Recent advances in cancer therapy targeting proteins involved in DNA double-strand break repair. Clin Cancer Res 15: 6314-6320, 2009.

18. Lehmann BD, McCubrey JA, Jefferson HS, Paine MS, Chappell WH and Terrian DM: A dominant role for p53-dependent cellular senescence in radiosensitization of human prostate cancer cells. Cell Cycle 6: 595-605, 2007.

19. Bergkvist GT, Argyle DJ, Pang LY, Muirhead R and Yool DA Studies on the inhibition of feline EGFR in squamous cell carcinoma: Enhancement of radiosensitivity and rescue of resistance to small molecule inhibitors. Cancer Biol Ther 11: 927-937, 2011.

20. Baumann M, Krause M and Hill R: Exploring the role of cancer stem cells in radioresistance. Nat Rev Cancer 8: 545-554, 2008.

21. Brown JM and Giaccia AJ: The unique physiology of solid tumors: Opportunities (and problems) for cancer therapy. Cancer Res 58: 1408-1416, 1998.

22. Milosevic M, Warde P, Menard C, Chung P, Toi A, Ishkanian A McLean M, Pintilie M, Sykes J, Gospodarowicz M, et al: Tumor hypoxia predicts biochemical failure following radiotherapy for clinically localized prostate cancer. Clin Cancer Res 18: 2108-2114, 2012

23. Harada H: Hypoxia-inducible factor 1-mediated characteristic features of cancer cells for tumor radioresistance. J Radiat Res (Tokyo) 57 (Suppl 1): i99-i105, 2016.

24. Sattler UG and Mueller-Klieser W: The anti-oxidant capacity of tumour glycolysis. Int J Radiat Biol 85: 963-971, 2009.

25. Trachootham D, Alexandre J and Huang P: Targeting cancer cells by ROS-mediated mechanisms: A radical therapeutic approach? Nat Rev Drug Discov 8: 579-591, 2009.

26. Bui T and Thompson CB: Cancer's sweet tooth. Cancer Cell 9: 419-420, 2006

27. Nakashima RA, Paggi MG and Pedersen PL: Contributions of glycolysis and oxidative phosphorylation to adenosine 5'-triphosphate production in AS-30D hepatoma cells. Cancer Res 44: 5702-5706, 1984.

28. Meng MB, Wang HH, Guo WH, Wu ZQ, Zeng XL, Zaorsky NG, Shi HS, Qian D, Niu ZM, Jiang B, et al: Targeting pyruvate kinase M2 contributes to radiosensitivity of non-small cell lung cancer cells in vitro and in vivo. Cancer Lett 356B: 985-993, 2015.

29. Bol V, Bol A, Bouzin C,Labar D, Lee JA,Janssens G, Porporato PE, Sonveaux P, Feron O and Grégoire V: Reprogramming of tumor metabolism by targeting mitochondria improves tumor response to irradiation. Acta Oncol 54: 266-274, 2015.

30. Pena-Rico MA, Calvo-Vidal MN, Villalonga-Planells R, Martínez-Soler F, Giménez-Bonafé P, Navarro-Sabaté À, Tortosa A, Bartrons R and Manzano A: TP53 induced glycolysis and apoptosis regulator (TIGAR) knockdown results in radiosensitization of glioma cells. Radiother Oncol 101: 132-139, 2011.

31. BelAiba RS, Djordjevic T, Bonello S, Flügel D, Hess J, Kietzmann $\mathrm{T}$ and Görlach A: Redox-sensitive regulation of the HIF pathway under non-hypoxic conditions in pulmonary artery smooth muscle cells. Biol Chem 385: 249-257, 2004.

32. Chandel NS, McClintock DS, Feliciano CE, Wood TM, Melendez JA, Rodriguez AM and Schumacker PT: Reactive oxygen species generated at mitochondrial complex III stabilize hypoxia-inducible factor-1alpha during hypoxia: A mechanism of $\mathrm{O}_{2}$ sensing. J Biol Chem 275: 25130-25138, 2000.
33. Cheng Y, Chen G, Hong L, Zhou L, Hu M, Li B, Huang J, Xia L and Li C: How does hypoxia inducible factor- $1 \alpha$ participate in enhancing the glycolysis activity in cervical cancer? Ann Diagn Pathol 17: 305-311, 2013.

34. Zhang Z, Zhou D, Lai Y, Liu Y, Tao X, Wang Q, Zhao G, Gu H, Liao H, Zhu Y, et al: Estrogen induces endometrial cancer cell proliferation and invasion by regulating the fat mass and obesityassociated gene via PI3K/AKT and MAPK signaling pathways. Cancer Lett 319: 89-97, 2012

35. Salani B, Del Rio A, Marini C, Sambuceti G, Cordera R and Maggi D: Metformin, cancer and glucose metabolism. Endocr Relat Cancer 21: R461-R471, 2014.

36. Salani B, Marini C, Rio AD, Ravera S, Massollo M, Orengo AM, Amaro A, Passalacqua M, Maffioli S, Pfeffer U, et al: Metformin impairs glucose consumption and survival in Calu-1 cells by direct inhibition of hexokinase-II. Sci Rep 3: 2070, 2013.

37. Semenza GL: Hypoxia-inducible factor 1 (HIF-1) pathway. Sci STKE 2007: $\mathrm{cm} 8,2007$.

38. Roberts DJ, Tan-Sah VP, Smith JM and Miyamoto S: Akt phosphorylates HK-II at Thr-473 and increases mitochondrial HK-II association to protect cardiomyocytes. J Biol Chem 288: 23798-23806, 2013.

39. Marini C, Salani B, Massollo M, Amaro A, Esposito AI, Orengo AM, Capitanio S, Emionite L, Riondato M, Bottoni G, et al: Direct inhibition of hexokinase activity by metformin at least partially impairs glucose metabolism and tumor growth in experimental breast cancer. Cell Cycle 12: 3490-3499, 2013

40. Peng G-Q, Yang Y, Zhong C-G, Yin H, Hu G and Tian Y: A study of association between expression of hOGG1, VDAC1, HK-2 and cervical carcinoma. J Exp Clin Cancer Res 29: 129, 2010.

41. Jin Z, Gu J, Xin X, Li Y and Wang H: Expression of hexokinase 2 in epithelial ovarian tumors and its clinical significance in serous ovarian cancer. Eur J Gynaecol Oncol 35: 519-524, 2014.

42. Huang X, Liu M, Sun H, Wang F, Xie X, Chen X, Su J, He Y, Dai $\mathrm{Y}, \mathrm{Wu} \mathrm{H}$, et al: $\mathrm{HK} 2$ is a radiation resistant and independent negative prognostic factor for patients with locally advanced cervical squamous cell carcinoma. Int J Clin Exp Pathol 8: 4054-4063, 2015.

43. Husseinzadeh $\mathrm{N}$ and Husseinzadeh HD: mTOR inhibitors and their clinical application in cervical, endometrial and ovarian cancers: A critical review. Gynecol Oncol 133: 375-381, 2014.

44. Li Y, Padmanabha D, Gentile LB, Dumur CI, Beckstead RB and Baker KD: HIF- and non-HIF-regulated hypoxic responses require the estrogen-related receptor in Drosophila melanogaster. PLoS Genet 9: e1003230, 2013

45. Fraga A, Ribeiro R and Medeiros R: Tumor hypoxia: The role of HIF. Actas Urol Esp 33: 941-951, 2009 (In Spanish).

46. Pitroda SP, Wakim BT, Sood RF, Beveridge MG, Beckett MA, MacDermed DM, Weichselbaum RR and Khodarev NN: STAT1dependent expression of energy metabolic pathways links tumour growth and radioresistance to the Warburg effect. BMC Med 7: 68, 2009.

47. Hirschhaeuser F, Sattler UG and Mueller-Klieser W: Lactate: A metabolic key player in cancer. Cancer Res 71: 6921-6925, 2011.

48. Shimura T, Noma N, Sano Y, Ochiai Y, Oikawa T, Fukumoto M and Kunugita N: AKT-mediated enhanced aerobic glycolysis causes acquired radioresistance by human tumor cells. Radiother Oncol 112: 302-307, 2014

49. Moeller BJ, Richardson RA and Dewhirst MW: Hypoxia and radiotherapy: Opportunities for improved outcomes in cancer treatment. Cancer Metastasis Rev 26: 241-248, 2007.

50. Cullis PM, Jones GD, Symons MC and Lea JS: Electron transfer from protein to DNA in irradiated chromatin. Nature 330: 773-774, 1987.

51. Bhatt AN, Chauhan A, Khanna S, Rai Y, Singh S, Soni R, Kalra N and Dwarakanath BS: Transient elevation of glycolysis confers radio-resistance by facilitating DNA repair in cells. BMC Cancer 15: 335, 2015

52. Pathania D, Millard M and Neamati N: Opportunities in discovery and delivery of anticancer drugs targeting mitochondria and cancer cell metabolism. Adv Drug Deliv Rev 61: 1250-1275, 2009.

53. Deng Q, Yu X, Xiao L, Hu Z, Luo X, Tao Y, Yang L, Liu X, Chen H, Ding Z, et al: Neoalbaconol induces energy depletion and multiple cell death in cancer cells by targeting PDK1PI3-K/Akt signaling pathway. Cell Death Dis 4: e804, 2013.

54. Roden $\mathrm{R}$ and Wu TC: How will HPV vaccines affect cervical cancer? Nat Rev Cancer 6: 753-763, 2006.

55. Doorbar J: Molecular biology of human papillomavirus infection and cervical cancer. Clin Sci (Lond) 110: 525-541, 2006. 
56. Münger K, Basile JR, Duensing S, Eichten A, Gonzalez SL, Grace $M$ and Zacny VL: Biological activities and molecular targets of the human papillomavirus E7 oncoprotein. Oncogene 20: 7888-7898, 2001.

57. Rodolico V, Arancio W, Amato MC, Aragona F, Cappello F, Di Fede O, Pannone G and Campisi G: Hypoxia inducible factor-1 alpha expression is increased in infected positive HPV16 DNA oral squamous cell carcinoma and positively associated with HPV16 E7 oncoprotein. Infect Agent Cancer 6: 18, 2011.

58. Bodily JM, Mehta KP and Laimins LA: Human papillomavirus E7 enhances hypoxia-inducible factor 1-mediated transcription by inhibiting binding of histone deacetylases. Cancer Res 71: $1187-1195,2011$

59. Nakamura M, Bodily JM, Beglin $M$, Kyo S, Inoue $M$ and Laimins LA: Hypoxia-specific stabilization of HIF-1alpha by human papillomaviruses. Virology 387: 442-448, 2009.

60. Li G, He L, Zhang E, Shi J, Zhang Q, Le AD, Zhou K and Tang X: Overexpression of human papillomavirus (HPV) type 16 oncoproteins promotes angiogenesis via enhancing HIF-1 $\alpha$ and VEGF expression in non-small cell lung cancer cells. Cancer Lett 311: $160-170,2011$
61. Gao JL and Chen YG: Natural compounds regulate glycolysis in hypoxic tumor microenvironment. BioMed Res Int 2015: 354143, 2015.

62. Marín-Hernández A, Gallardo-Pérez JC, Ralph SJ, RodríguezEnríquez S and Moreno-Sánchez R: HIF-lalpha modulates energy metabolism in cancer cells by inducing over-expression of specific glycolytic isoforms. Mini Rev Med Chem 9: 1084-1101, 2009.

63. Wang L, Xiong H, Wu F, Zhang Y, Wang J, Zhao L, Guo X, Chang LJ, Zhang Y, You MJ, et al: Hexokinase 2-mediated Warburg effect is required for PTEN- and p53-deficiency-driven prostate cancer growth. Cell Rep 8: 1461-1474, 2014.

64. Zhuo B, Li Y, Li Z, Qin H, Sun Q, Zhang F, Shen Y, Shi Y and Wang R: PI3K/Akt signaling mediated Hexokinase-2 expression inhibits cell apoptosis and promotes tumor growth in pediatric osteosarcoma. Biochem Biophys Res Commun 464: 401-406, 2015. 